Gastroenterologe 2012 · 7:6-7

DOI 10.1007/s11377-011-0574-5

Online publiziert: 12. Januar 2012

(c) Springer-Verlag 2012

R.M. Schmid • J.F. Riemann ${ }^{2}$

${ }^{1}$ 2. Medizinische Klinik, Klinikum rechts der Isar, Technische Universität München

${ }^{2} \mathrm{c} / \mathrm{o}$ Stiftung LebensBlicke, Klinikum Ludwigshafen

\title{
Personalisierte Tumortherapie
}

die Therapie des metastasierten Magenkarzinoms qualifiziert.

Frau Prof. Reinacher-Schick diskutiert die Datengrundlage für die risikoadaptierte adjuvante Therapie des Kolonkarzinoms. Während die Leitlinie der DGVS beim Befall regionärer Lymphknoten (UICC III) den Empfehlungsgrad A für die Chemotherapie mit Folfox oder Capox ausspricht, hat diese Therapie im Stadium II auch bei Patienten mit Risikofaktoren keinen Stellenwert. Liegen Risikofaktoren vor, sollte eine adjuvante Therapie mit 5-FU erfolgen. Für Patienten ohne Risikofaktoren besteht eine „Kann“-Empfehlung. Eine Besonderheit beim Kolonkarzinom sind Patienten mit hochgradiger Mikrosatelliteninstabilität. Diese Tumoren unterscheiden sich bezüglich der genetischen Veränderungen von Tumoren, die mikrosatellitenstabil sind. Tumoren mit hochgradiger Mikrosatelliteninstabilität weisen eine höhere Infiltrationsdichte mit Lymphozyten auf und haben eine deutlich günstigere Prognose. Eine MSI-Untersuchung könnte daher diese Subgruppe des Kolonkarzinoms im Stadium II identifizieren.

\section{I) Entscheidend sind Biomarker mit prädiktivem Wert für den Therapienutzen}

In der metastasierten Situation des Kolonkarzinoms ist die personalisierte Therapie heutzutage schon etabliert. Für die Therapieentscheidung müssen KRAS-Mutationen bestimmt und Mutationen in weiteren Signalmolekülen wie PI3-Kinase und $B R A F$ analysiert werden. Dies erläutert Herr Dr. Ettrich in seinem Artikel.
GIST-Tumoren sind im Gegensatz $\mathrm{zu}$ den anderen gastrointestinalen Tumoren monogenetische Erkrankungen mit aktivierenden Mutationen in der Stammzellfaktor-Rezeptortyrosinkinase oder der PDGFR- $\alpha$-Kette. Herr Priv.-Doz. von Bubnoff bespricht in seinem Artikel aktuelle Therapieansätze mit Tyrosinkinase-Inhibitoren.

Entscheidend für die personalisierte Therapie sind Biomarker mit prädiktivem Wert für den Nutzen eines bestimmten Therapieprotokolls. Es geht darum vorauszusagen, ob das Medikament wirkt oder nicht. Darüber hinaus kann die wirkungslose Therapie für den Patienten schädlich sein. Ein weiterer Aspekt sind die Therapiekosten.

Wir stehen mit diesen Möglichkeiten ganz am Anfang. Wir sind davon überzeugt, dass mit Hilfe von HochdurchsatzSequenzierungsverfahren das Tumorgenom in wenigen Jahren routinemäßig vor der Therapie analysiert werden wird. Die Kosten für die Analyse der Genome werden im Vergleich zu den Therapiekosten verschwindend gering sein. Die erfassbare Heterogenität in den einzelnen Entitäten wird deutlich zunehmen, damit wird die Gruppengröße der Subentitäten kleiner. Prospektive randomisierte Phase-IIIStudien werden dann schwieriger durchführbar sein. Da die Zulassungsbehörden weiterhin sehr harte Kriterien an die Einführung neuer Medikamente stellen werden, bedarf es einer besseren Vernetzung, um diese wichtigen Studien durchführen zu können.

Wir glauben, dass der Gastroenterologe die Prinzipien der Molekular- und Zellbiologie mit der Aufdeckung zell- und gewebsspezifischer Genexpressionsmus- 
ter, die ja nicht nur für die Tumortherapie, sondern auch für wichtige andere Bereiche wie $z$. B. die Behandlung der Hepatitis $C$ gelten, kennen und einordnen lernen sollte. Die Beiträge dieses Heftes zeigen einmal mehr, wie Grundlagenforschung diagnostische und therapeutische Konzepte beeinflussen und entscheidende Weichenstellungen in deren Weiterentwicklung bewirken kann.

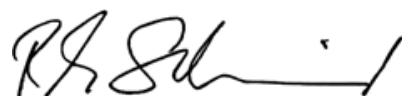

Roland M. Schmid

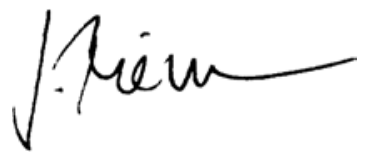

Jürgen F. Riemann

\section{Korrespondenzadressen}

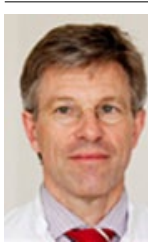

Prof. Dr. R.M. Schmid

2. Medizinische Klinik,

Klinikum rechts der Isar, Technische Universität München Ismaninger Straße 22, 81675 München Roland.Schmid@ Irz.tu-muenchen.de

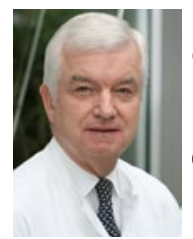

Prof. Dr. J.F. Riemann c/o Stiftung LebensBlicke, Klinikum Ludwigshafen Bremserstraße 79, 67063 Ludwigshafen riemannj@garps.de

\section{Literatur}

1. Roychowdhury S, lyer MK, Robinson DR et al (2011) Personalized oncology through integrative high-throughput sequencing: a pilot study. SciTransl Med 3: 111 ra121

\title{
30. Deutscher Krebskongress
}

\author{
Berlin, 22. - 25. Februar 2012 \\ Entwicklungen in der Krebsforschung aus erster Hand
}

\begin{abstract}
Die steigende Zahl der Krebsneuerkrankungen ist alarmierend. Nur bessere Versorgungsstrukturen und optimierte Therapien können in dieser Situation weiterhelfen. Dazu braucht es eine exzellente Forschung und eine hohe Qualität in der Patientenversorgung. Um diesen Erfordernissen Nachdruck zu verleihen, wurde der kommende Deutsche Krebskongress unter das Motto „Qualität sichern - Forschung fo(e)rdern" gestellt. Denn Forschungsförderung sollte an wesentlichen Punkten durchaus auch politisch eingefordert werden.
\end{abstract}

\section{Besonderheiten des DKK 2012}

- Neben der Präsentation herausragender wissenschaftlicher Beiträge konzentriert sich das Programm auf gesundheitspolitische Themen. Im Rahmen der DKK-Veranstaltungen zum Nationalen Krebsplan werden Themen wie Krebsfrüherkennung, Qualitätssicherung, Arzneimittelbewertung und Patienteninformation diskutiert. Darüber hinaus wird es ein Symposium zur Qualität in der Patientenversorgung geben, das erstmalig in der Geschichte des DKK gemeinsam mit der renommierten amerikanischen Gesellschaft für klinische Onkologie ASCO durchgeführt wird. Symposien zur Forschungsförderung, zur Betreuung Langzeitüberlebender mit
Krebs und zu Krebs bei älteren Patienten runden das breite Spektrum der gesundheitspolitischen Veranstaltungen des DKK 2012 ab.

\section{Interdisziplinär und International}

- Der DKK zeichnet sich vor allem durch seine interdisziplinäre Ausrichtung aus - die klinische Onkologie ist dort ebenso vertreten wie die Radiotherapie, die onkologische Chirurgie, die Palliativmedizin und die onkologische Pflege. Um die Diskussion der vorgestellten Ergebnisse mit renommierten Kollegen aus dem In- und Ausland zu fördern, wurden außerdem neue Formate wie thematisch zusammenhängende Plenarsitzungen mit international besetzten „Key-Note-Lectures", „best-of"-Sitzungen und moderierte Diskussionsrunden ins Programm aufgenommen.

\section{Weitere Informationen}

- Das DKK-Vorprogramm kann ab sofort heruntergeladen werden: www krebsgesellschaft.de/dkk_2012_programm.

- Unter folgendem Link können Sie einen Newsletter zum DKK 2012 abonnieren: www.krebsgesellschaft.de/dkk_2012_presseverteiler 